\title{
Effect of Character Animacy and Preparatory Motion on Perceptual Magnitude of Errors in Ballistic Motion
}

\author{
P. S. A. Reitsma ${ }^{1}$, J. Andrews ${ }^{2}$, and N. S. Pollard ${ }^{3}$ \\ ${ }^{1}$ Trinity College Dublin, Ireland \\ ${ }^{2}$ University of California, Berkeley, USA \\ ${ }^{3}$ Carnegie Mellon University, USA
}

\begin{abstract}
An increasing number of projects have examined the perceptual magnitude of visible artifacts in animated motion. These studies have been performed using a mix of character types, from detailed human models to abstract geometric objects such as spheres. We explore the extent to which character morphology influences user sensitivity to errors in a fixed set of ballistic motions replicated on three different character types. We find user sensitivity responds to changes in error type or magnitude in a similar manner regardless of character type, but that users display a higher sensitivity to some types of errors when these errors are displayed on more human-like characters. Further investigation of those error types suggests that being able to observe a period of preparatory motion before the onset of ballistic motion may be important. However, we found no evidence to suggest that a mismatch between the preparatory phase and the resulting ballistic motion was responsible for the higher sensitivity to errors that was observed for the most humanlike character.
\end{abstract}

Categories and Subject Descriptors (according to ACM CCS): I.3.7 [Computer Graphics]: Three-Dimensional Graphics and Realism

\section{Introduction}

A substantial body of recent work has examined the problem of quantifying the perceptual magnitude of errors in animated motion. While data on a variety of errors and scenarios has been generated, these results come from studies

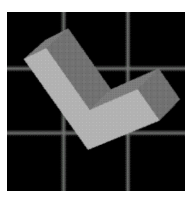

(a)

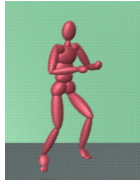

(b)

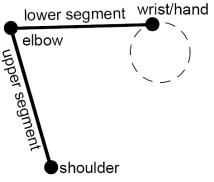

(c)

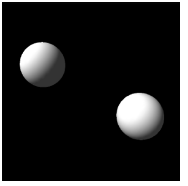

(d)
Figure 1: A selection of the character types used in recent perceptual studies of animation. (a) O'Sullivan et al. [ODGK03] (b) Reitsma and Pollard [RP03] (c) Harrison et al. [HRvdP04] (d) O'Sullivan and Dingliana [OD01]

(c) 2008 The Author(s)

Journal compilation (c) 2008 The Eurographics Association and Blackwell Publishing Ltd. Published by Blackwell Publishing, 9600 Garsington Road, Oxford OX4 2DQ, UK and 350 Main Street, Malden, MA 02148, USA using a wide array of different character types, from human figures of varying realism to abstract geometric objects such as circles (Figure 1). Unfortunately, the character differences make comparing results between these studies very difficult, as the interaction between character type and user sensitivity to error is largely unknown.

There is some evidence that improved graphical quality of animations may increase the ability of users to detect anomalous motions (e.g., [SW93] [HOT98] [OHJ00] [HB00]). In particular, Hodgins and her colleagues [HOT98] demonstrated that subjects are more sensitive to motion changes displayed on polygonal humanoid characters as compared to humanoid characters formed by stick figures. The reason for this difference is unknown, however; one hypothesis is that the more realistic polygonal character allows greater sensitivity than the simpler and more abstract stick figure.

We explore this hypothesis, considering the extreme difference between a human character and a sphere. In particular, we ask whether the findings of previous researchers are 


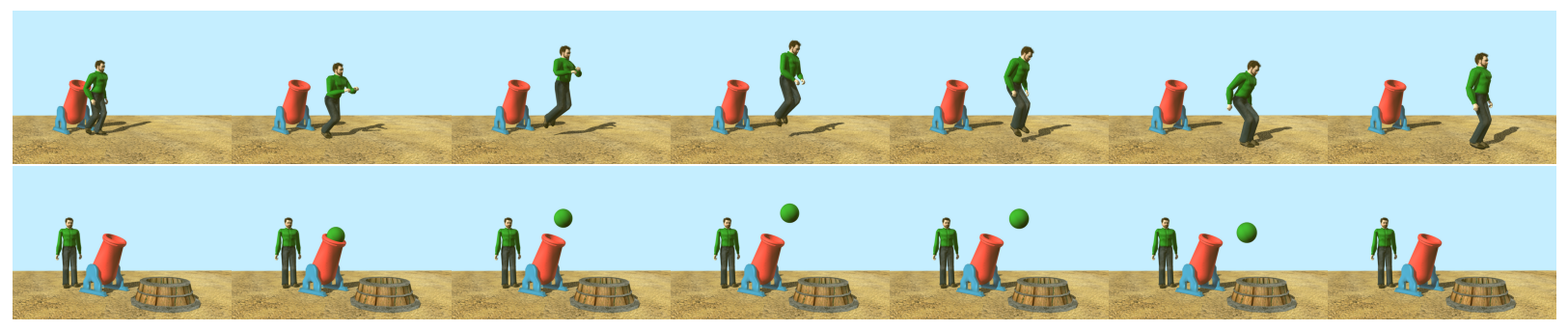

Figure 2: An example of one of the ballistic motions used in our user studies, depicted on two of the characters used: a human figure (top) and a cannonball (bottom) which followed the same center of mass trajectory. The second frame is the takeoff point where the character transitions into ballistic motion.

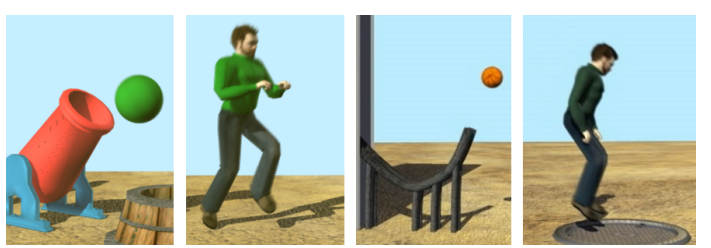

Figure 3: The set of characters employed in our user studies. Left two characters were used in the first study; right two characters were used in the second study.

replicated or contradicted in the case of ballistic motion. Consider a human character and a sphere, both of which undergo ballistic motion with identical center of mass trajectories (Figure 2). Intuitively, one might expect that errors in the ballistic trajectory would be easier to detect with the ball "character", due to the simplicity of its motion and the lack of distractions such as limb motion. Our familiarity with human motion, though, makes humans very skillful at detecting anomalies in human motion, suggesting that perhaps subjects would draw on their experience to display greater sensitivity to errors in the trajectory of the human character. Without some knowledge of how sensitivity to errors varies with character type, our ability to compare and exploit the results of studies performed on different characters is limited. Indeed, neuroimaging studies (e.g., [GDP*00] $\left.\left[\mathrm{PMM}^{*} 03\right]\right)$ suggest that different regions of the brain are active when observing coherent motion deemed biological or non-biological, suggesting that there may be fundamental and irreconcilable differences between user perceptions of animated motion in complex human characters and in simple, abstract objects such as spheres.

In this paper, we explore ballistic motions derived from human motion captured jumps. We add errors of three varieties, motivated by the types of errors that arise during motion editing and transitions between motions: a quick change in vertical velocity during the flight phase; a quick change in horizontal velocity (in the direction of the jump) during the flight phase; and a change in apparent gravity over the entire flight phase. We present full human body motion through a human character, and identical center of mass motion through a spherical object (Figure 3). Our primary motivation is to answer three questions:

1. Does a difference in user ability to detect added errors exist between realistic human characters and simple, abstract characters for the particular case of ballistic motion?

2. If a difference does exist, is it consistent? i.e., are the perceptual characteristics of motion on one character type reliably predictable from the perceptual characteristics of motion on the other character type?

3. If there is a consistent difference, how much and in what direction? i.e., are changes to ballistic motion easier to detect on a simple, abstract character on or a realistic human character?

Additionally, we examine potential underlying causes for the observed differences. In particular, we explore the effect of displaying a preparatory motion for the ball, as shown in Figure 6.

In summary, we find no difference in user ability to detect horizontal or vertical velocity errors regardless of character type, but we find that changes in the level of gravity are easier to detect with a human character than with a ball character. The sensitivity difference was smaller if a preparatory motion for the ball was displayed then when it was not; however, subjects were significantly more sensitive to motion of the human character in both cases. Perhaps surprisingly, there was no evidence to suggest that sensitivity was affected by a mismatch (a different gravitational constant) between the preparatory motion and the ballistic motion of the ball. In other words, it did not appear to matter whether the preparatory motion was consistent with the ballistic motion, only that a preparatory motion was present.

\section{Background}

A number of researchers have examined the perceptual magnitude of errors in motion. Most have examined the phenomenon using geometric objects such as circles or dots 
(e.g., [Mic63] [Coh64] [Run74] [SW93] [OD01]), spheres and rigid bodies [ODGK03], or two-link chains [HRvdP04], although recently some researchers have looked at human characters [RP03] [WB03] [WB04] [RPE* 05].

Researchers have also examined the effects on user perception of differences in character model or animation. Hodgins et al. [HOT98] showed that subjects are more sensitive to differences between pairs of motions when those motions are displayed on a polygonal character rather than a stick figure character; similarly, Chaminade et al. [CHK07] found that subject ability to discriminate between biological and non-biological motion was impaired when that motion was displayed on a character that was a cloud of points rather than a polygonal model character or even a character built up out of ellipses. Stappers and Waller [SW93] noted that more complex visual stimuli, such as more droplets in an animated fountain, made users more sensitive to depth estimation in a virtual scene. Oesker et al. [OHJ00] showed that greater detail and realism in the animation of soccer-playing humanoids allowed subjects to more accurately gauge the skill with which the humanoids were playing. All of these experiments indicate that character complexity can affect our ability to make judgements about motion; however, the effect of character differences on user sensitivity to physical errors in character motion has not yet been examined.

It is generally acknowledged that people perform poorly on abstract physical reasoning tasks [Pro99], although animation has been shown to improve performance [KPWH92]. Hecht and Bertamini [HB00] showed that subjects have a poor innate understanding of ballistic motion and were tolerant of substantial errors in ballistic trajectory for animations of thrown balls, including added accelerations and decelerations. Neuroimaging studies (e.g., [GDP*00] and $\left.\left[\mathrm{PMM}^{*} 03\right]\right)$, however, make it unclear whether the findings of Hecht et al. on simple projectiles apply to recognizablyhuman characters, although Chaminade et al. [CHK07] do not find a consistent difference in brain activity between character types.

Reitsma and Pollard [RP03] examined user sensitivity to errors in the ballistic phase of motion captured human jumping motions. We adopt identical techniques to examine the sensitivity differences of subjects to errors in motions displayed on rigid bodies vs. humanoid characters. As with their approach, we use detection theory [MC91] to convert rating data to sensitivity measures.

\section{Study 1: Effect of Character Animacy on Error Sensitivity}

Our first study was designed to answer our three core questions:

1. Are errors in ballistic motion more apparent with realistic human characters or with simple sphere "characters"?

2. If a difference does exist, is it consistent?
3. If there is a consistent difference, how much and in what direction?

We selected the experimental methodology of Reitsma and Pollard [RP03] for our experiment; one benefit of this choice was the ability to compare our two character models (realistic and abstract; see Figure 3) to their semi-realistic character model (Figure 1(b)), enhancing the discriminatory power of our experiment.

Our two characters, a simple ball and a human model, were chosen to represent two extremes in the range of possible characters, the ball being one of the simplest possible characters, with no moving parts, and the human being a complex, but intuitively understood, character.

Participants: Participants were obtained by university-wide advertising. 9 women and 13 men ranging in age from 18 to 33 successfully completed the study; four additional participants did not follow instructions and were excluded from the analysis.

Participants were excluded based on whether their responses on the exit questionnaire clearly revealed that they had not followed instructions. The most common reason for exclusion was a failure to follow the instruction that animations were to be judged based on their appearance while the character was in the air; for example, one participant was excluded for indicating that they based some of their decisions on how the human character behaved after finishing the landing phase of its jump.

Stimuli: Participants were shown animations of two characters - a textured human character and a spherical cannonball - undergoing ballistic motion. All animations were shown in the same rendering style, with the same (fixed) camera configuration (Figure 2), with the character beginning the motion at the same position and jumping in the same direction each time. Shadows were rendered, and a small amount of motion blur was added. These parameters were chosen so as to make this user study as comparable as possible to the studies performed by Reitsma and Pollard [RP03], as were other details of our experimental setup, such as user study conditions, participant instructions, camera angle, and apparent character size.

Animations of human jumping motions were created as stimuli using seven source motions, which were modified with three error varieties and three error magnitudes as listed in Table 1. Horizontal and vertical errors were created by smoothly adding the indicated level of velocity to the root of the character over a $0.1 \mathrm{~s}$ time window starting $0.1 \mathrm{~s}$ after the beginning of the ballistic phase of motion. Gravity errors were created by altering the level of gravity over the entire ballistic phase of the motion. For motions whose ballistic phase changed duration due to the added error, the non-root motion of the character was timewarped to fit the new duration of the ballistic phase, with linear interpolation between 


\begin{tabular}{|c|c|c|c|}
\hline Error & \multicolumn{3}{|c|}{ Error Magnitude } \\
Variety & Small & Medium & Large \\
\hline Horizontal & $\pm 0.45 \mathrm{~m} / \mathrm{s}$ & $\pm 0.73 \mathrm{~m} / \mathrm{s}$ & $\pm 1.10 \mathrm{~m} / \mathrm{s}$ \\
Vertical & $\pm 0.45 \mathrm{~m} / \mathrm{s}$ & $\pm 0.73 \mathrm{~m} / \mathrm{s}$ & $\pm 1.10 \mathrm{~m} / \mathrm{s}$ \\
Gravity & $\pm 1.70 \mathrm{~m} / \mathrm{s}^{2}$ & $\pm 2.70 \mathrm{~m} / \mathrm{s}^{2}$ & $\pm 4.00 \mathrm{~m} / \mathrm{s}^{2}$ \\
\hline
\end{tabular}

Table 1: Error magnitudes used for our first user study.

values at adjacent frames used to resample joint poses as needed. In all cases, character root velocity at the end of the ballistic phase was edited via displacement splines to match the root landing velocity in the original motion capture. These displacement splines were extended as far back into the ballistic motion as possible to avoid presenting a second source of perceptible errors. Source motions and error treatments were identical to those used in Reitsma and Pollard [RP03].

Motions for the ball character consisted of three parts linked together in a $\mathrm{C} 1$-continuous manner, corresponding to the takeoff, ballistic, and landing phases of the jumps performed by the human character. The takeoff phase for the ball consisted of smooth linear acceleration from a fullyhidden position inside the cannon's barrel to the point where the ball was half-emerged from the mouth of the cannon (first and second frame respectively of Figure 2). The ball then entered its ballistic phase, and followed the center of mass trajectory of the human character performing that motion (frames three through five of Figure 2). Finally, the frame where the human character's feet touch the ground marks the beginning of the landing phase; during this phase, the ballistic motion of the ball was extended by continuing its motion at constant gravitational acceleration until it entered a basket placed on the ground plane and disappeared from view (last two frames of Figure 2). In order to provide scale cues, the human character was placed beside the cannon, and remained motionless throughout the animation (Figure 3).

Procedure. Participants were told they would see two types of animated motions: a human jumping, or a ball traveling through similar arcs. They were given some background information on how motion capture data is created. Participants were told that half of the motions had errors, that errors were similar in each of the two types of animations, and that errors occurred during the flight phase of a motion.

Participants were then shown a training set of 24 motions, consisting of each of the two characters (human and ball) being used to display the same 12 representative animations. They were told that half contained errors but were not told which specific motions in this training set contained errors. Source motions for each error treatment were chosen at random, and the presentation order of the full set of 24 animations was randomly permuted. This training set allowed users to see motions with errors of all detectability levels, allowing them to calibrate their use of the 0-9 scale and make use of the full range of available responses. Not only did this help prevent a learning effect from skewing the initial responses, using the full range of the scale provides greater separation of the data, and hence more information on the sensitivity of the participant.

Each error treatment was shown on two different source motions, for 36 unique error-containing trials. The original source motion for each of these trials was also included, bringing the total to 72 motions. Finally, each motion was displayed on each of the two characters, for a total of 144 test motions. The order of presentation of the 144 animations was randomly permuted, and then split into four blocks of 36 trials for display purposes.

All motions were placed on DVD in movie format and played on a commercial DVD player, as that appeared to minimize playback hitches compared to playback from either a video file on computer or from a DVD in a computer DVD drive. Six DVDs were used, each with a different random assignment of source motions to error treatments, and each with a unique permutation of presentation order for the resulting animations. Each DVD was seen by between 3 and 5 subjects.

Stimuli were presented on a projection screen in a small conference room. Participants were instructed to categorize each motion as either "no error (unchanged)" or "error" and mark their level of confidence in their answer using a rating scale that ranged from 0 (most confident an error is present) through 9 (most confident an error is not present). Participants categorized training motions as well as test motions, but the data from the training motions was not used for any analyses.

At the end of the study, participants were asked to describe their experience in the study of motion, including involvement in sports, dance, video games, etc.

\subsection{Detection Theory}

As motion capture data provides only a lossy approximation of the original human motion, all user judgements will be inherently subjective. Accordingly, substantial response bias is possible; for example, one respondent might think all motion captured data looks poor, and hence rate all motions with a low score, whereas another respondent might like animated motion and tend to rate all motions highly. Unless taken into account by the analysis technique, such between-subject differences would dilute the findings of the experiment.

As noted by Reitsma and Pollard [RP03], detection theory [MC91] can be used to derive a bias-independent measure of a user's ability to detect errors in an animated motion. The method takes into account the difference between how frequently the subject correctly labelled a motion as contain- 
ing an error (hit rate $H$ ) and how frequently the subject incorrectly labelled an unchanged motion as containing an error (false alarm rate $F$ ). For the simplest experimental design (a yes/no response), a subject's sensitivity $(d)$ to errors would be computed as:

$$
d=z(H)-z(F)
$$

where $z$ is the inverse of the normal distribution function. For example, a hit rate of $75 \%$ and a false alarm rate of $25 \%$ corresponds to a sensitivity of 1.35 , as does a hit rate of $90 \%$ coupled with a false alarm rate of just over $47 \%$. These two examples of how to obtain a sensitivity of 1.35 demonstrate the bias-independent nature of detection theory: as sensitivity is computed based on the relative distribution of the participant's responses rather than on the raw distribution, factors which will systematically bias the responses, such as participant reaction to the quality of the animation, are automatically factored out.

A similar approach allows sensitivity levels to be computed from rating data (intuitively, a high mean rating corresponds to a high hit rate and high false alarm rate; see [MC91] for details).

\subsection{Study 1 Results}

Figure 4 shows mean sensitivities for all error varieties and directions for both characters used in our first user study. The plots show the mean sensitivities of motions at each magnitude of added error, including unchanged motions. Mean values from Reitsma and Pollard's 2003 paper [RP03] are plotted for comparison, and are directly comparable.

We used the residual maximum likelihood (REML) method (see [PT71] [CS76]) to analyze the per-subject sensitivity values (computed as per [MC91]) with 3 error levels x 2 character types (human and ball) x 3 error varieties x 2 error directions. This technique was chosen due to our use of a mixed model with subject ID as a random effect; this design allowed inter-subject variability to be taken into account more explicitly. All error varieties could be detected with $P<0.01$, and no effect of experience $(F(1,736)=1.88, P=$ $0.17)$ or gender $(F(1,736)=0.56, P=0.46)$ was found.

We found that error type affected perceptual differences between different characters:

(1) Subjects found gravity errors easier to detect with human characters than with ball characters

$F(1,233)=5.98, P=0.015$

(2) Subjects displayed no significant difference in ability to detect either horizontal or vertical errors regardless of whether human characters or ball characters were used $F(1,233)=2.03, P=0.16$ and $F(1,233)=0.002, P=0.97$, respectively

Our study gave very comparable overall results to the studies reported by Reitsma and Pollard [RP03]. Our study confirmed the three main effects found in their work:

(RP1) Subjects found added acceleration easier to detect than added deceleration

Human: $F(1,238)=44.33, P=0.001$

Ball: $F(1,238)=15.85, P<0.001$

(RP2) Subjects found low gravity easier to detect than high gravity

Human: $F(1,107)=32.48, P<0.001$

Ball: $F(1,107)=13.09, P<0.001$

(RP3) Subjects found errors in horizontal velocities easier to detect than errors in vertical velocities

Human: $F(1,238)=20.13, P<0.001$

Ball: $F(1,238)=25.57, P<0.001$

Sensitivity to horizontal and vertical errors did not differ significantly between the studies $(F(1,53)=1.64, P=$ $0.20)$. Sensitivity to gravity errors did not differ significantly between the previous study and our study's human character $(F(1,31)=3.58, P=0.068)$ or between the previous study and our study's ball character $(F(1,31)=3.82, P=0.060)$. This result is especially interesting in light of the substantial difference between our human and ball characters (main effect 1); one possible explanation is that perhaps the rough human figure used by Reitsma and Pollard may have been "perceptually in between" our realistic human character and simple ball character.

\section{Study 2: Effect of Preparatory Motion}

Study 1 identified no significant difference in user sensitivity between character types for the case of vertical or horizontal velocity perturbations, but did identify a substantial difference for the case of changes to gravity over the entire ballistic phase of the motion (see Section 3.2). The goal of our second study was to investigate possible mechanisms responsible for this difference. In particular, our intent was to control for possible sources of systematic bias in the experimental design to narrow down what factors caused the observed difference. Possible sources of systematic bias include:

1. Mismatch between preparatory and ballistic motion only for the human character.

2. Signalling of landing position only for the ball character.

3. Inherent scale cues only for the human character.

In Study 2, we correct these three differences and further investigate the first of these differences as a possible cause of the results in Study 1.

In Study 1, human character animation contained more information in the form of preparatory motion. In particular, the "apparent effort" of a human preparing for a jump could be observed in the human motions, whereas the ball 

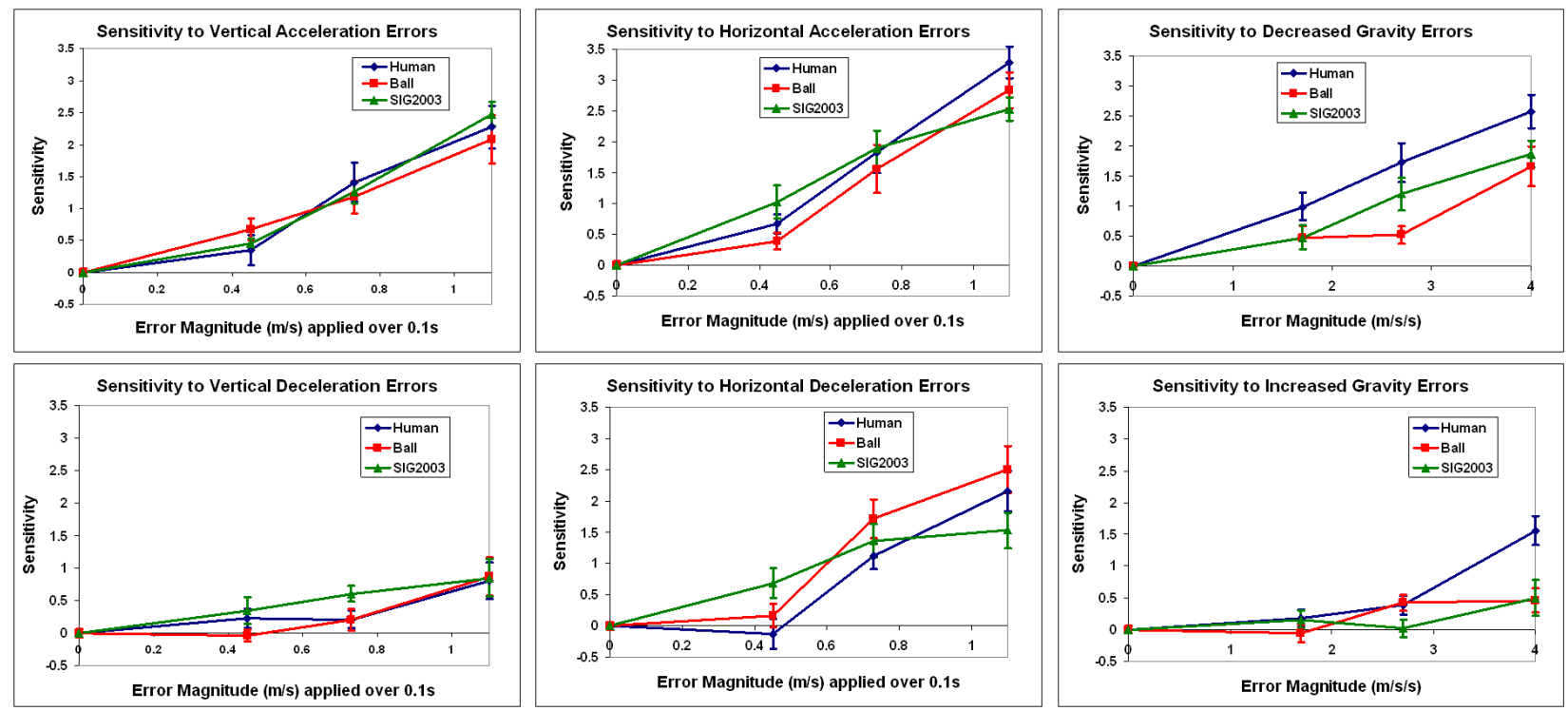

Figure 4: Mean sensitivities for all errors from both characters used in the first study, as well as the character from Reitsma and Pollard [RP03]. Error bars show standard error of the mean.

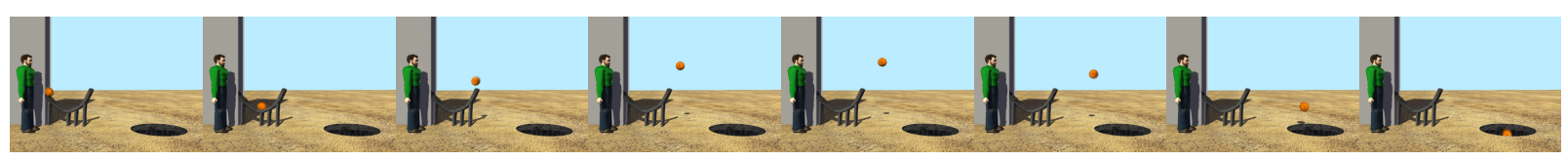

Figure 5: An example of the preparatory and ballistic motion seen in our second user study. The character transitions to ballistic motion just prior to frame 3 .

displayed no "preparatory motion", as it was launched from a cannon. A mismatch between the perceived effort and the actual height or distance of a jump was hypothesized to explain the difference in sensitivity.

To test this hypothesis, in the new study, the ball character was given preparatory motion; i.e., shown rolling along a launch ramp before undergoing ballistic motion (Figure 5). Though a number of different launch devices, such as a spring, a rubber band, or a 'pushing rod,' could be used to explain the ball's launch velocity, rolling the ball down the ramp without introducing any external launch devices was chosen as the clearest, simplest method for generating the preparatory motion. In particular, it did not introduce any additional moving parts to the animation, and the correct effect of gravity may be intuitively understood.

To remove the second identified source of bias, both human and ball characters had their landing points marked in a similar manner (a manhole cover for the human to land on and an equivalent-sized hole for the ball to vanish into). Finally, we addressed the question of inherent scale cues by using a new ball character with the size and textured appearance of a basketball (see Figure 6). As with the first experiment, the human character was positioned beside the launch apparatus to provide additional scale cues.

Participants: Participants were obtained by university-wide advertising. 9 women and 10 men ranging in age from 18 to 32 successfully completed the study; three additional subjects were excluded from the analysis due to hardware failure during their session.

Stimuli: Participants were shown animations of two characters - a textured human character and a textured spherical ball - undergoing preparatory and then ballistic motion. The experimental setup was similar to the previous study, but was changed to accommodate the launch ramp and larger error magnitudes (Table 2); accordingly, character textures, camera angle, and camera distance from the characters had slightly different (fixed) values.

This study used the same source ballistic motions as the previous study, and applied gravity errors in the same man- 


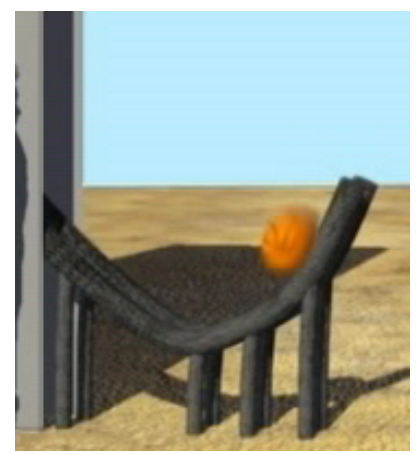

Figure 6: Experimental setup used to display preparatory motion for the ball character in the second study. The ball rolls along the launch ramp under either the correct gravitational force or under a gravitational force consistent with the following ballistic motion.

\begin{tabular}{|c|c|c|c|}
\hline Error & \multicolumn{3}{|c|}{ Error Magnitude } \\
Direction & Small & Medium & Large \\
\hline Decreased & $\pm 2.7 \mathrm{~m} / \mathrm{s}^{2}$ & $\pm 4.0 \mathrm{~m} / \mathrm{s}^{2}$ & $\pm 5.5 \mathrm{~m} / \mathrm{s}^{2}$ \\
Increased & $\pm 3.4 \mathrm{~m} / \mathrm{s}^{2}$ & $\pm 5.4 \mathrm{~m} / \mathrm{s}^{2}$ & $\pm 8.0 \mathrm{~m} / \mathrm{s}^{2}$ \\
\hline
\end{tabular}

Table 2: Gravity error magnitudes used for our second user study. Magnitudes were based on pretesting with the new experimental setup.

ner; prior to undergoing ballistic motion, three cases of preparatory motion were used:

- Correct: the ball accelerated according to normal gravity (i.e., $9.8 \mathrm{~m} / \mathrm{s}^{2}$ ).

- Consistent: the ball accelerated according to the level of gravity present in the ballistic motion.

- Hidden: the ball could not be seen prior to ballistic motion.

The Correct case corresponds to the situation for the human character: since the human is animated using the untouched motion capture data during the takeoff phase of the jump, it is subject to the correct level of gravity during that takeoff, resulting in an acceleration mismatch between preparatory and ballistic motion. By contrast, the Consistent case has no mismatch between the level of gravity experienced by the ball during preparatory and ballistic motion; gravity is consistent through all phases of the motion. Finally, the Hidden case corresponds to the cannonball character from the first study, as the preparatory motion can not be seen.

\section{Procedure:}

Participants were given similar instructions to the previous study, were shown 20 representative training examples, and then were shown 144 test motions. The experimental apparatus (projection screen, DVD playback, response sheets, etc.) was identical to the previous study. Each test consisted
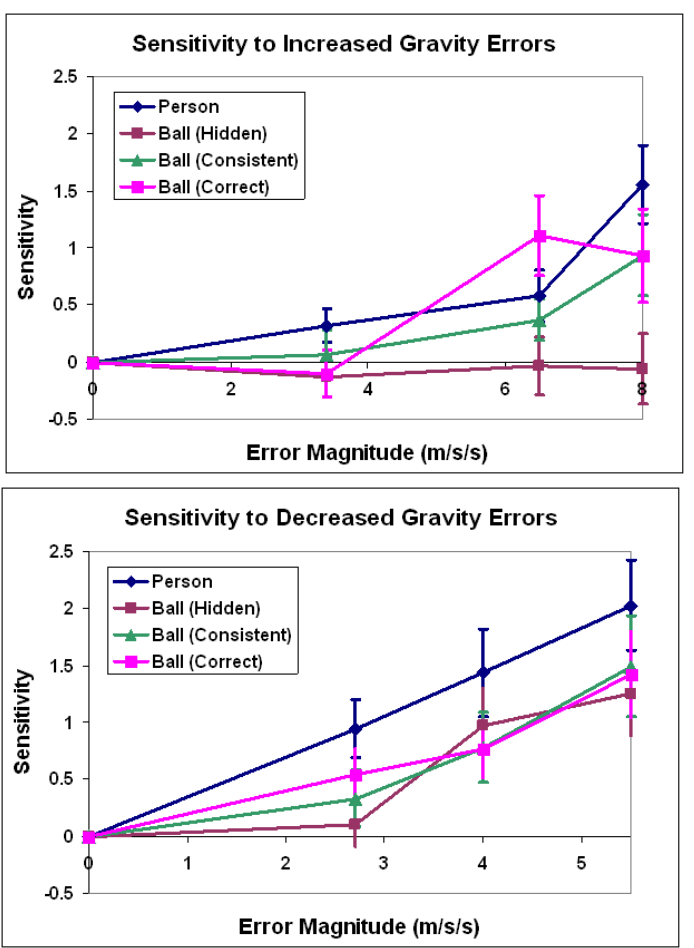

Figure 7: Mean sensitivities for all character types used in our second study. Error bars show standard error of the mean.

of 36 unique motions -3 examples of each error treatment shown on three different source motions, plus the corresponding error-free motions - displayed on each of the four characters, for a total of 144 test motions. The order of presentation of the 144 animations was randomly permuted, and then split into four blocks of 36 trials for display purposes.

Four DVDs were used, each with a different random assignment of source motions to error treatments, and each with a unique permutation of presentation order for the resulting animations. Each DVD was seen by between 4 and 5 subjects.

\subsection{Study 2 Results}

Figure 7 shows mean sensitivities for all character types and both directions used in our second user study. Results are broken out by small, medium, and large error levels.

As with the first experiment, we used the REML method to analyze the per-subject sensitivity values, with 3 error levels x 4 character treatments x 2 error directions. All error varieties could be detected with $P<0.01$ except for increased gravity errors in the Hidden case, and no effect of experience $(F(1,418)=0.25, P=0.62)$ or gender $(F(1,418)=$ $0.21, P=0.65)$ was found. 
The second study produced three main effects:

(1) Subjects found gravity errors easier to detect when displayed on a human character with preparatory motion than when displayed on a ball character with preparatory motion.

$F(1,426)=5.58, P=0.019$.

(2) Subjects found increased gravity errors displayed on ball characters easier to detect when shown preparatory motion than when they were shown no preparatory motion

$F(1,336)=6.01, P=0.015$.

(3) Subjects showed no significant difference in sensitivity when preparatory motion for the ball character was computed with normal gravity or with gravity consistent with the (altered) ballistic phase of the motion.

$F(1,222)=0.41, P=0.52$.

In addition to the first main effect, relating the Consistent and Correct cases of the ball character to the human case, subjects also found errors easier to detect on the human than on the ball in the Hidden case $(F(1,227)=18.94, P<$ $0.001)$. Sensitivity to the human character was significantly different from sensitivity to any case of the ball character (Student's $t=1.97, \alpha=0.05$ ). Sensitivity was significantly higher to decreased gravity on the human character than on any other condition, and was significantly lower to increased gravity in the Hidden case than for any other condition, but there was insufficient data to further discriminate between $\{$ Type $\mathrm{x}$ Direction $\}$ conditions.

\section{Discussion}

In these studies, we measured sensitivity of human subjects to errors in animated ballistic human and ball motion. We found that changes in the level of gravity were easier to detect with a human character than with a ball character, but that there was no significant difference in sensitivity between human characters and ball characters for horizontal or vertical errors. Similarly, we found that changes in the level of gravity displayed on a ball character were easier to detect when shown preparatory motion, but that the correctness of the preparatory motion did not make a significant difference, and showing the preparatory motion did not erase the sensitivity difference between human and ball characters.

Based on these results, we can address our motivating questions from Section 1:

1. Does a difference exist?

2. Is the difference consistent?

3. In which direction is the difference?

Our results indicate that perceptual differences exist between the sensitivity of users to changes in motions displayed on abstract ball or realistic human characters, but only for some types of changes. For our case of ballistic motion, the sensitivity of users to local perturbations in the horizontal or vertical center of mass velocity did not differ between the characters tested; i.e., the difference was consistent, and approximately zero. This result stands in apparent conflict with the results of Hodgins et al. [HOT98] and Chaminade et al. [CHK07], and further research is needed to understand under what circumstances character type will affect our ability to judge the realism of motion. One possible explanation is that simple and abrupt changes to the character's root motion, as may result from splicing and editing operations, may be less influenced by character type than the more complex motion modifications explored in those two prior studies. Indeed, anecdotal evidence from our poststudy questionnaire suggested that subjects tended to detect horizontal and vertical errors via direct observation of their effects, whereas gravity errors, which had less abrupt and more complex effects, were detected via observation of their effect on the overall character of the motion.

Changes in the level of gravity did result in significantly different levels of sensitivity for the different characters. This difference in user sensitivity between characters was consistent for decreases in gravity, with subjects showing approximately twice the sensitivity to decreased gravity displayed on the human character as on the ball character. By contrast, the sensitivity difference between characters was not consistent for increases in gravity, with sensitivity on the more realistic character appearing to rise much more rapidly with large error magnitudes.

Our second study examined the differences in sensitivity to altered gravity in more detail, removing several possible sources of bias and testing the hypothesis that the higher sensitivity found in the first study for the human character is explained by a mismatch between preparatory motion and subsequent ballistic motion; in essence, that the apparent effort of the character was often too much or too little for the magnitude of the following jump.

Our results showed that the presence and correctness of preparatory motion made no difference for user sensitivity to decreased gravity displayed on the ball character. All cases of preparatory motion resulted in perceptual magnitudes which were lower than for the human character by roughly $30 \%$; however, the sensitivity difference varied substantially from that mean difference in the case where the preparatory motion was hidden.

By contrast, each preparatory motion treatment of the ball character resulted in qualitatively different user sensitivities for increased gravity errors. The ball displayed with preparatory motion consistent with its ballistic motion showed the same pattern as before: user sensitivity consistently lower than the human character by roughly $30 \%$. However, despite the lack of significant difference in sensitivity between the two types of preparatory motion, there was a qualitative difference for increased gravity. User sensitivity to the ball dis- 
played with "correct" preparatory motion (i.e., whose gravity was always $9.8 \mathrm{~m} / \mathrm{s}^{2}$ regardless of gravity during the ballistic phase) resulted in perceptual magnitudes for the gravity increases which did not differ by a consistent amount from the results for the human character, but which were qualitatively similar to those for the cannonball in the first study: i.e., relatively high sensitivity to medium errors as compared to sensitivity to small and large errors.

Finally, users had no sensitivity to increased gravity errors displayed on the ball character whose preparatory motion was hidden. One likely factor contributing to this lack of sensitivity is that motions with increased gravity and hidden preparatory motion were visible for only a brief time; when gravity was increased by the maximum amount, the longest of these motions was visible for $20 \%$ less time than the shortest motion for which preparatory motion was shown. There is no indication from the results, however, that difference in motion duration between trials within a single error treatment has an effect on the score assigned by subjects. The raw source motions had flight phase durations which differed by up to $20 \%$, but no effect of flight phase duration was found among the scores given to error-free motions in the second study as a whole $(F(1,2718)=1.40, P=0.24)$, or to motions whose preparatory motion was hidden $(F(1,679)=$ $0.01, P=0.92)$. Accordingly, it appears as though raw duration of a motion may not fully explain the low user sensitivity to errors in cases with hidden preparatory motion.

In all cases, user sensitivity to gravity errors displayed on the human character was significantly higher than user sensitivity to errors displayed on the ball character, suggesting factors other than those controlled for in the second experiment must account for a significant portion of the difference. One possibility is that because gravity errors correspond to incorrect behavior over the entire jump, rather than a localized disruption, subjects' greater familiarity with human jumping motion than ball trajectories would account for the difference, especially because it is known that humans have a poor intuitive sense for the physics of the ballistic trajectories of simple objects such as balls [HB00]. Another possibility is that because a change in the force of gravity corresponds to timescaling of the jump, the animated motions of the limbs and head of the human character offered additional information that was not present in the ball character. Stappers and Waller [SW93] note that richer stimuli improved accuracy and reliability in using the free fall of objects under gravity to estimate visual depth, suggesting that the additional information offered by the limbs of the human character may have improved user results in our experiment. They also note how observed gravity can vary with perceived scale and distance, suggesting the possibility that the human jumping innately embodies a sense of scale, whereas subjects may have some freedom to interpret the size and distance of the cannonball to suit the observed motion, notwithstanding the human figure placed beside the cannon and the use of a clearly identified basket- ball for the spherical object in the second study. Finally, it is worth noting that observing biological motion engages our mirror neurons [GDP $\left.{ }^{*} 00\right]\left[\mathrm{PMM}^{*} 03\right]$ - allowing us to internally mimic an observed motion - in a way non-biological motion does not. One speculative but interesting possibility is that this neurological difference makes humans inherently more sensitive to certain types of errors in human motion than in motion perceived as non-biological or inanimate.

\section{Conclusions}

This work examined the extent to which the animacy and human-like nature of a character affects user sensitivity to errors in ballistic motion, and the effect of displaying matched, mismatched, or no preparatory motion for simple characters.

Our experiments demonstrated that user sensitivity to errors in ballistic motion follows the same general linear pattern for characters ranging between a human and a ball, suggesting that it is not unreasonable to attempt to generalize results and findings from one character type to another. Substantial care must be taken with such generalizations, however, as our experiments also demonstrated that for some types of errors subjects tend to be more sensitive to motion displayed on human characters than on rough human figures or simple geometric objects. Whether or not preparatory motion matches ballistic motion appears to play a limited role in this difference. As ballistic motion is substantially simpler than general motion, due to the lack of ground contacts, this result suggests animators may not need to aggressively extend edits in ballistic motion into the ground contact phases before and after, potentially saving substantial effort.

Additionally, the low user sensitivity to increased gravity in the case with hidden preparatory motion may indicate that fast, smooth motions which are visible for only a short time may not allow a user to fully evaluate their physical plausibility, and hence may look acceptable even with substantially larger errors. As rapid motion out of concealment is a common scenario is applications such as real-time computer games, we are interested in further exploring the extent to which brief, unexpected, or partially-occluded motions may be able to absorb higher levels of error for the same loss of perceptual quality than slower, longer, and more expected motions.

Finally, we note that the error types for which character morphology made no difference to user sensitivity (added velocity in the horizontal or vertical direction) are inherently local changes to the motion; subject responses to the post-study questionnaire suggested that these types of motions were typically detected by direct observation of their effects (e.g., the character was "pushed", or the motion was jerky). By contrast, the errors for which user sensitivity differed significantly between the character types (increased or decreased gravity) are global changes which extend over a much wider duration of the underlying motion, and sub- 
ject responses suggested that these types of errors were typically detected by indirect observation (e.g., the character "floated", or travelled "too far"). Intuitively, we would expect a subject's greater familiarity with human motion to assist their detection when motions are detected indirectly by their effect on the overall character of the motion, but to have a more limited effect when errors are detected by direct observation. We are interested in determining whether this local/global split is an artifact of the particular errors we tested, or whether it might apply more generally.

\section{Acknowledgements}

Supported in part by the IRCSET Embark Initiative and by the NSF under grants ISS-0326322, CCF-0343161, and ECS-0325383. Alias/Wavefront donated their Maya software for use in this research. The authors would like to thank Raphael Mun, Daniel Hill, and Moshe Mahler for their assistance with this work.

\section{References}

[CHK07] Chaminade T., Hodgins J., Kawato M.: Anthropomorphism influences perception of computeranimated characters' actions. Social Cognitive and Affective Neuroscience, 2(3); p.206-216 (2007).

[Coh64] Cohen R. L.: Problems in motion perception. Uppsals, Sweden: Lundequistska (1964).

[CS76] Corbeil R. R., SEARLE S. R.: Restricted maximum likelihood (reml) estimation of variance components in the mixed model. Technometrics, Vol 18., No. 1 (1976).

[GDP*00] Grossman E., Donnelly M., Price R., Pickens D., Morgan V., Neighbor G., Blake R.: Brain areas involved in perception of biological motion. Journal of Cognitive Neuroscience, 12:5, pp. 711-720 (2000).

[HB00] Hecht H., Bertamini M.: Understanding projectile acceleration. Journal of Experimental Psychology: Human Perception and Performance 26, 2 (2000), 730746.

[HOT98] Hodgins J. K., O’Brien J. F., Tumblin J.: Perception of human motion with different geometric models. IEEE Transactions on Visualization and Computer Graphics 4, 4 (October 1998), 307-316.

[HRvdP04] HARRISON J., RENSINK R. A., VAN DE PANNE M.: Obscuring length changes during animated motion. ACM Transactions on Graphics 23, 3 (Aug. 2004), 569-573.

[KPWH92] Kaiser M. K., Proffitt D. R., Whelan S. M., HECHT H.: Influence of animation on dynamical judgments. Journal of Experimental Psychology: Human Perception and Performance 18, 3 (1992), 669-690.
[MC91] Macmillan N. A., Creelman C. D.: Detection Theory: A User's Guide. Cambridge University Press, New York, 1991.

[Mic63] Мichotте A.: The perception of causality. Metheun, London (1963).

[OD01] O'Sullivan C., Dingliana J.: Collisions and perception. ACM Transactions on Graphics 20, 3 (July 2001), 151-168.

[ODGK03] O'Sullivan C., Dingliana J., Giang T., KAISER M. K.: Evaluating the visual fidelity of physically based animations. In ACM Transactions on Graphics 2, 3 (2003).

[OHJ00] Oesker M., Hecht H., Jung B.: Psychological evidence for unconscious processing of detail in realtime animation of multiple characters. Journal of Visualization and Computer Animation 11 (2000), 105-112.

[PMM*03] Pelphrey K. A., Mitchell T. V., MCKeown M. J., Goldstein J., Allison T., McCarthy G.: Brain activity evoked by the perception of human walking: Controlling for meaningful coherent motion. The Journal of Neuroscience, 23(17):6819-6825 (2003).

[Pro99] ProffitT D. R.: Naive physics. In The MIT Encyclopedia of the Cognitive Sciences, Wilson R., Keil F., (Eds.). MIT Press, 1999, pp. 577-579.

[PT71] PATTERSON H., ThOMPSON R.: Recovery of inter-block information when block sizes are unequal. Biometrika 58, p.545-554 (1971).

[RP03] Reitsma P. S. A., Pollard N. S.: Perceptual metrics for character animation: Sensitivity to errors in ballistic motion. ACM Transactions on Graphics 22, 3 (July 2003), 537-542.

[RPE* 05] REN L., PATrick A., EFros A. A., Hodgins J. K., REHG J. M.: A data-driven approach to quantifying natural human motion. ACM Transactions on Graphics 24, 3 (Aug. 2005), 1090-1097.

[Run74] RUNESON S.: Constant velocity - not perceived as such. Psychological Research, 37(1), pp. 3-23 (1974).

[SW93] Stappers P. J., WALleR P. E.: Using the free fall of objects under gravity for visual depth estimation. Bulletin of the Psychonomic Society 31, 2 (1993), 125127.

[WB03] WANG J., Bodenheimer B.: An evaluation of a cost metric for selecting transitions between motion segments. In 2003 ACM SIGGRAPH / Eurographics Symposium on Computer Animation (Aug. 2003), pp. 232-238.

[WB04] Wang J., BodenheImer B.: Computing the duration of motion transitions: an empirical approach. In 2004 ACM SIGGRAPH / Eurographics Symposium on Computer Animation (July 2004), pp. 335-344. 